\title{
Association between Malondialdehyde, GSH/GSSG Ratio and Bone Mineral Density in Postmenopausal Women
}

\author{
Innawati Jusup ${ }^{1}$, Lusiana Batubara1, Dwi Ngestiningsih¹, Faizah Fulyani ${ }^{1}$, Devina Afraditya Paveta², \\ Putri Theresia Lemmo Ate Bancin² \\ ${ }^{1}$ Department of Medical Biology and Biochemistry, Faculty of Medicine, Universitas Diponegoro, Semarang, Indonesia \\ ${ }^{2}$ Undergraduate Program, Faculty of Medicine, Diponegoro University, Semarang, Indonesia
}

Background: Osteoporosis is one of chronic degenerative diseases especially in postmenopausal women, characterized by a decreased bone mass due to imbalance activity between osteoblasts and osteoclasts. Recently, oxidative stress is believed to play an important role in osteoporosis pathogenesis. Oxidative stress is commonly considered as the consequence of an imbalance between pro and antioxidants species, which results in damage in the affected tissue. Malondialdehyde (MDA) is frequently used as a biomarker of oxidative stress in many health problems since MDA is produced at high levels during lipid peroxidation. Meanwhile, glutathione is well known as one of antioxidant which against oxidative stress by preserving its homeostasis in the reduced form of glutathione sulfhydryl (GSH) and the oxidized form of glutathione disulphide (GSSG). This study was aimed to determine the association between MDA, GSH/GSSG ratio and bone mineral density (BMD) in postmenopausal women.

Materials and method: We conducted a cross-sectional study in 40 postmenopausal women. MDA and GSH/GSSG ratio were assessed by enzyme-linked immunosorbent assay (ELISA). Bone mineral density (BMD) was obtained from secondary data. The statistical analysis was conducted using Spearman rho's correlation test.

Results: Based on the test, we didn't found significant correlation between MDA and BMD ( $r=-0.054, p=0.741)$, but we found significant moderate correlation between GSH/GSSG ratio ( $r=0.436, p=0.005)$ and BMD in postmenopausal women. Conclusion: There was no correlation between MDA and BMD in postmenopausal women. However, there was significant moderate correlation between GSH/GSSG ratio and BMD in postmenopausal women.

Keywords: MDA, GSH/GSSG ratio, BMD, osteoporosis

\section{Introduction}

Osteoporosis has been known as the silent epidemic disease. As affecting the bone tissue and may causing long term injury due to fracture, osteoporosis constitutes a major health problem, especially in postmenopausal women. ${ }^{1}$
International Osteoporosis Foundation claimed 1 out of 4 women in a range of 50-80 years old in Indonesia has increased risk of osteoporosis. Osteoporosis is caused by imbalance activity of osteoblasts (bone formation cells) and osteoclasts (bone resorption cells) which destroy microarchitecture within the trabecular of bone. ${ }^{2}$

Date of submission: January 11, 2020

Last Revised: July 6, 2020

Accepted for publication: August 7, 2020

Corresponding Author:

Innawati Jusup

Department of Medical Biology and Biochemistry, Faculty of Medicine

Universitas Diponegoro, Jl. Prof. Soedarto, Tembalang

Semarang, Jawa Tengah 50275, Indonesia

e-mail: innawati.jusup@fk.undip.ac.id

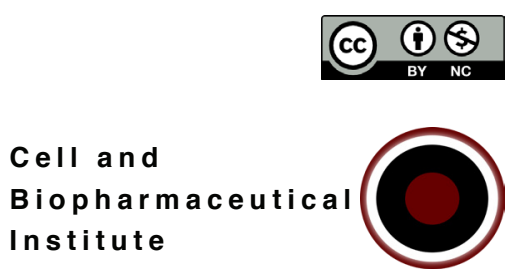


Bone tissue can be normally affected by various factors, such as oxygen supplies, nutrition, hormones, cytokines, growth factors, and oxidative stress. Recently, some studies indicating that oxidative stress plays important role in osteoporosis pathogenesis. Under oxidative stress condition, high circulating free radicals will destruct the bone tissue. Therefore, osteoblasts will produce antioxidants in order to protect bone cells from injury caused by free radicals. ${ }^{3,4}$

Lipid peroxidation causes damage to cells in the body, such as mitochondria, DNA, lipids, and proteins, which then leads to the production of some reactive aldehydes. ${ }^{5}$ In degenerative diseases such as osteoporosis, which is characterized by increased oxidative stress, malondialdehyde (MDA) plays an important pathogenic role, since it is able to form bonds with proteins, such as cell apoptosis thus causing functional damage and accelerating molecular turnover. ${ }^{6}$ MDA is commonly used as a biomarker of oxidative stress in many health problems such as osteoporosis, cancer, asthma, or cardiovascular disease because MDA is produced at high levels during lipid peroxidation. MDA levels can be measured in serum, plasma and tissue samples, using various methods. ${ }^{7}$

Some studies showed decreasing amount of glutathione peroxidase and glutathione reductase in osteoporosis patients. Glutathione peroxidase is an enzyme which catalyses reduction of free radical by producing glutathione disulphide (GSSG) or oxidized form of glutathione. Meanwhile, glutathione reductase is an enzyme that uses GSSG to produce glutathione sulfhydryl (GSH) or reduced form of glutathione. Concentration ratio of GSH and GSSG can be assessed as the GSH/GSSG ratio and represented the oxidative stress level in the tissue. ${ }^{4}$

The gold standard for diagnosing osteoporosis is by examining the bone mineral density (BMD) at a certain part of the bone, such as femoral neck or lumbar vertebral bone. The BMD score obtained from the dual energy x-ray scan (DXA scan) will be calculated with formula to get the T-score of the patient. ${ }^{2}$ This study was aimed to determine the association between MDA, GSH/GSSG ratio and BMD in postmenopausal women.

\section{Materials and methods}

\section{Subjects and Ethical Statements}

This was a observational cross-sectional study, which was conducted in 40 postmenopausal women in Semarang
Elderly Integrated Healthcare Center (Genuksari, Tegalsari, and Sanggung Raya), Indonesia. The inclusion criteria were postmenopausal women who had normal menopause history and was able to communicate well. Subjects with chronic diseases (diabetes mellitus, renal failure, and chronic heart disease), long term corticosteroid treatment, long term calcium, and vitamin D supplements consumption were excluded from the study. Demographic data of study population were recorded.

The study protocol was approved by the Ethics Committee of Faculty of Medicine, Universitas Diponegoro (ethical clearance No. 209/EC/KEPK/FK-UNDIP/V/2019). Before participating in the study, all participants already signed the written informed consents.

\section{BMD Measurement}

BMD of subjects were measured at the femoral neck and lumbar vertebrae areas by DXA scans. To examine the BMD of the femoral neck, the patients were asked to lie on their backs, with toes together, and heels separated for $23 \mathrm{~cm}$. To examine the BMD of the lumbar vertebrae, the patients were asked to lie on their backs with knees flexed over a padded box to flatten the lumbar lordosis. BMD status was then assessed by T-score based on WHO criteria of osteoporosis. T-score $\geq-1$ was classified as normal BMD, T-score between $<-1$ and $>-2.5$ was classified as osteopenia, and T-score $\leq-2.5$ was classified as osteoporosis. All BMD data were acquired from secondary data.

\section{MDA Measurement}

Plasma MDA was determined by enzyme-linked immunosorbent assay (ELISA)-competitive principle (Cat No. E-EL-0060, Elabscience, Texas, USA). The ELISA microplate provided in this kit has been pre-coated with MDA. During the reaction, MDA in the sample or standard competes with a fixed amount of MDA on the solid phase supporter for sites on the Biotinylated Detection Ab specific to MDA. Excess conjugate and unbound sample or standard were washed from the plate, and Avidin conjugated to Horseradish Peroxidase (HRP) are added to each microplate well and incubated. Then, TMB substrate solution was added to each well. The enzyme-substrate reaction was terminated by the addition of stop solution. The optical density (OD) of all specimens were measured at $450 \mathrm{~nm}$ with microplate reader. The concentration of MDA in the samples is then determined by comparing the OD of the samples to the standard curve. 


\section{GSH/GSSG Ratio Measurement}

GSH/GSSG ratio was measured by the cyclic reaction of 5,5'-dithiobis-2-nitrobenzoic acid (DTNB) using colorimetric method with ELISA Total Glutathione/ Oxidized Glutathione Assay Kit (Cat No. E-BC-K097, Elabscience). The collected blood specimen from subjects were centrifugated to gain the blood plasma. Ten $\mu \mathrm{L}$ of blood plasma were mixed with $100 \mu \mathrm{L}$ of working reagents (WR)$1,10 \mu \mathrm{L}$ of WR-2, and $50 \mu \mathrm{L}$ of WR-3 to measure the total glutathione (T-GSH). To measure the GSSG concentration, $100 \mu \mathrm{L}$ of blood plasma were prepared through the pretreatment steps by mixing it with $2 \mu \mathrm{L}$ of WR-5 and $5 \mu \mathrm{L}$ of WR- 6 before incubated in $37^{\circ} \mathrm{C}$ for 30 minutes. Ten $\mu \mathrm{L}$ of pre-treatment solution were mixed with $100 \mu \mathrm{L}$ of WR$1,10 \mu \mathrm{L}$ of the WR-2, and $50 \mu \mathrm{L}$ of the WR-3. The optical density (OD) of all specimens were measured at $405 \mathrm{~nm}$ with microplate reader at 30 seconds (A1) and 630 seconds (A2), respectively, with $\Delta \mathrm{A}=\mathrm{A} 2-\mathrm{A} 1$.

\section{Statistical Analysis}

Software Statistical Package Sciences (SPSS) version 23.00 (IBM Corporation, New York, USA) was used to analyze the data. Saphiro-wilk test was performed to test the normality of data, while Spearman's rho correlation coefficients were performed to test the bivariate correlation.

\section{Results}

The baseline characteristic of the study population showed that the mean of age was $64.28 \pm 4.51$ years old, the mean of body weight was $55.88 \pm 8.98 \mathrm{~kg}$, the mean of body height was $1.50 \pm 0.05$ meter, the mean of BMI was $24.60 \pm 4.00$ $\mathrm{kg} / \mathrm{m}^{2}$, the mean of plasma MDA levels was $310.73 \pm 73.80$ $\mathrm{ng} / \mathrm{mL}$, the mean of GSH/GSSG ratio was $58.70 \pm 196.00$,

Table 1. Baseline characteristic of study populations.

\begin{tabular}{lc}
\hline \multicolumn{1}{c}{$\begin{array}{c}\text { Variables } \\
(\mathbf{n}=\mathbf{4 3})\end{array}$} & Mean \pm SD \\
\hline Age (year) & $64.28 \pm 4.51$ \\
Body weight $(\mathrm{kg})$ & $55.88 \pm 8.98$ \\
Body height $(\mathrm{m})$ & $1.50 \pm 0.05$ \\
BMI $\left(\mathrm{kg} / \mathrm{m}^{2}\right)$ & $24.60 \pm 4.00$ \\
Plasma MDA levels $(\mathrm{ng} / \mathrm{mL})$ & $310.73 \pm 73.80$ \\
GSH/GSSG ratio & $58.70 \pm 196.00$ \\
BMD $\left(\mathrm{g} / \mathrm{cm}^{2}\right)$ & $-2.56 \pm 1.10$ \\
\hline
\end{tabular}

the mean of BMD were $-2.56 \pm 1.10 \mathrm{~g} / \mathrm{cm}^{2}$ (Table 1). Plasma MDA levels were not significantly correlated to BMD score ( $p=0.741$ ), but GSH/GSSG ratio were found significantly correlated to BMD score $(p=0.004)$ (Table 2$)$.

\section{Discussion}

Oxidative stress is defined as an imbalance condition between antioxidants and pro-oxidants, usually indicated by low antioxidants level and increased oxidative stress markers. Lipid peroxidation is one of the most harmful effects of ROS, of which MDA is the end product, and it was seen that serum levels of MDA increased in bone disorders. Decreased activities of antioxidant enzymes superoxidase dismutase (SOD) and Glutathione peroxidase (GSH-PX) explain a defense mechanism that may have been overcome in alleviating the increased of superoxide production by osteoclasts displayed by increased levels of MDA in the serum. Antioxidants are generally believed to be protective against oxidative stress by several mechanisms. ${ }^{7}$

This study analyzed the relationship of plasma MDA levels to BMD, but there was no significant relationship between the two variables. The results of this study were consistent with supporting previous research, which stated that there was no correlation between MDA levels among osteoporosis and non-osteoporosis patients. ${ }^{8}$ Therefore, MDA levels may not help in determining the postmenopausal osteoporosis severity.

Compared to former study which obtained a correlation between glutathione peroxidase enzyme and BMD in postmenopausal women, in the present study, we found that there was significant correlation between GSH/GSSG ratio and BMD score in postmenopausal women. Glutathione and glutathione-related-enzyme work in cooperation to scavenge free radicals such as reactive oxygen species (ROS) and/or neutralize their toxic oxidizing effect. Glutathione peroxidase enzyme itself is an enzyme that

Table 2. Correlation between plasma MDA levels, GSH/ GSSG ratio and BMD score (T-score femur).

\begin{tabular}{lcc}
\hline \multirow{2}{*}{ Variables } & \multicolumn{2}{c}{ BMD } \\
\cline { 2 - 3 } & $\mathbf{r}$ & $\boldsymbol{p}$-value \\
\hline Plasma MDA levels & -0.054 & 0.741 \\
GSH/GSSG ratio & 0.43 & $0.004^{*}$ \\
\hline
\end{tabular}

*Spearman's rho test with significance at the level of 0.05 . 
catalyzes reduction process of ROS such as $\mathrm{H}_{2} \mathrm{O}_{2}$ become water molecule and change the reduced form of glutathione (GSH) into an oxidized form of glutathione (GSSG) by binding disulphide molecule., ${ }^{4,8}$

For oxidation-reduction reactions involving GSH and GSSG in which the reaction needs $2 \mathrm{e}^{-}$transfer so it requires 2 GSH to form 1 GSSG. Normally, GSH/GSSG ratio in the tissue is preserved in high level as a part of body mechanism to minimize the accumulation of intracellular disulphide concentration that may give toxic effect to the tissue. When chronic oxidative stress attacked certain tissue, as proposed in osteoporosis pathogenesis, it would lead to buffer changes through large increasing GSSG levels due to the inability of cells to reduce GSSG back to GSH as effectively normal., ${ }^{9,10}$ Thus, GSH/GSSG ratio would decrease as well. This might explain why there was significant correlation between GSH/ GSSG ratio and BMD in this study (Figure 1).

Some limitations of our study should be acknowledged. First, we couldn't control the variety of subject's weight, BMI, and diet. Secondly, we didn't assess any other oxidative stress biomarker. Researchers used MDA as an oxidative stress biomarker, which has disadvantages such as its unstable nature, especially if the examination is delayed for some time from sampling, thus researchers tried to save the delayed sample with ice box immediately after sampling and kept it on $-70^{\circ} \mathrm{C}$. MDA also has no normal value and is not specific to a particular disease. The variety of MDA examination methods is also related to the specificity of the results of the examination.

\section{Conclusion}

There was no significant correlation between plasma MDA levels and BMD in postmenopausal women. Besides, there was significant moderate correlation between GSH/GSSG ratio and BMD in postmenopausal women. Therefore, this study could be a cornerstone for further study related to $\mathrm{GSH} / \mathrm{GSSG}$ ratio examination and osteoporosis in postmenopausal women.

\section{References}

1. Kementerian Kesehatan RI. Pedoman Pengendalian Osteoporosis Menteri Kesehatan Republik Indonesia. Keputusan Menteri Kesehat Nomor 1142/MENKES/SK/XII/2008. Jakarta; Kementrian Kesehatan RI; 2008.

2. Szulc P, Bouxsein M. Vertebral Fracture Initiative Part 1. Overview of Osteoporosis: Epidemiology and Clinical Management. Cambridge: International Osteoporosis Foundation; 2011.

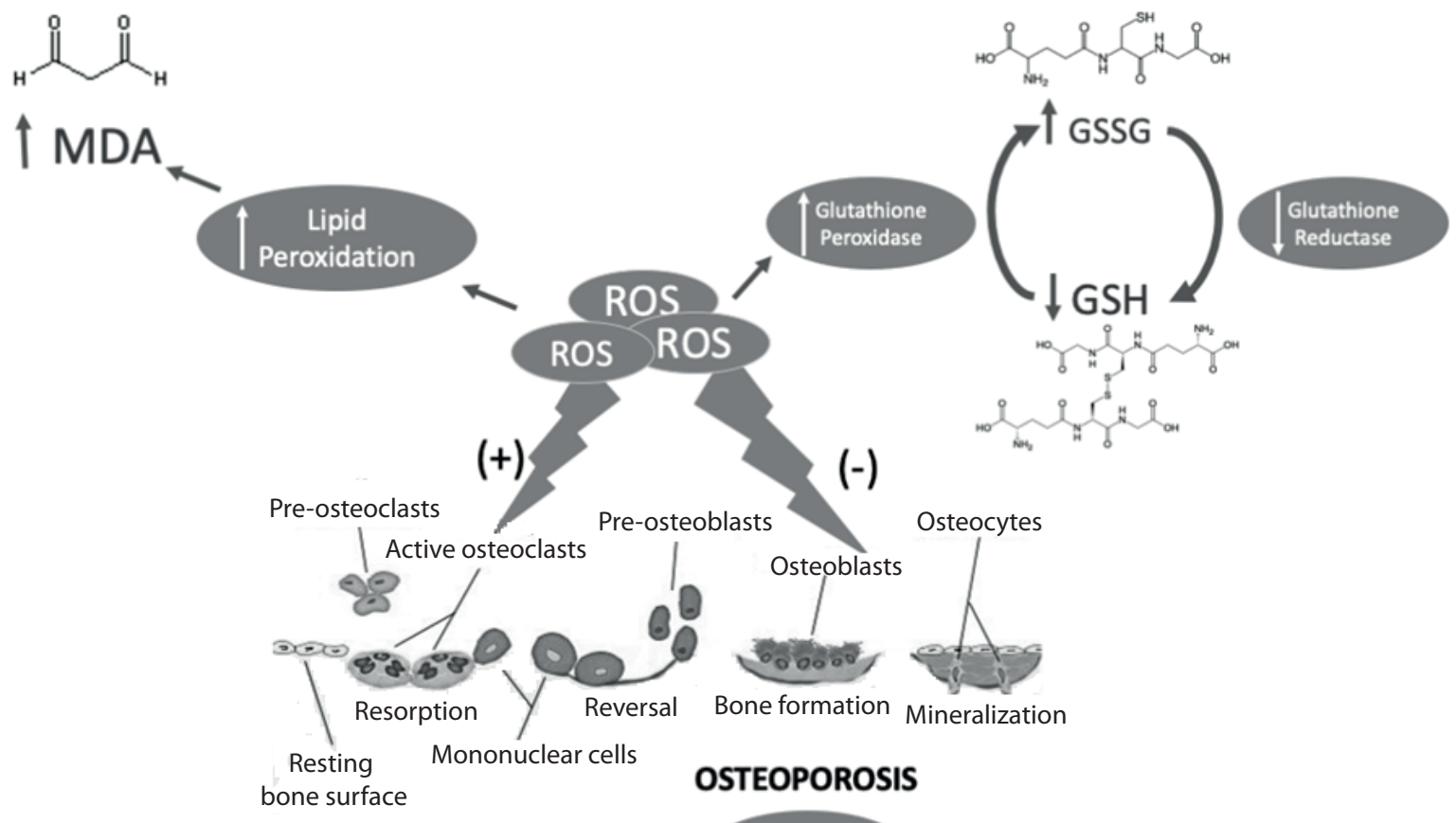

BMD

Figure 1. Relationship of MDA, GSH/GSSG Ratio and BMD. MDA: malondialdehyde; ROS: reactive oxygen species; GSSG: glutathione disulphide; GSH: glutathione sulfhydryl; BMD: bone mineral density. 
3. Mlakar SJ, Osredkar J, Prezelj J, Marc J. The antioxidant enzyme GPX1 gene polymorphisms are associated with low BMD and increased bone turnover markers. Dis Markers. 2010; 29(2): 71-80.

4. Lushchak VI. Glutathione homeostasis and functions: potential targets for medical interventions. J Amino Acids. 2012; 2012: 1-26. doi: $10.1155 / 2012 / 736837$.

5. Barrera G, Pizzimenti S, Daga M, Dianzani C, Arcaro A. Lipid peroxidation-derived aldehydes, 4-hydroxynonenal and malondialdehyde in aging-related disorders. Antioxidants. 2018; 7(8): 102. doi: 10.3390/antiox7080102.

6. Domazetovic V, Marcucci G, Iantomasi T, Brandi ML, Vincenzini MT. Oxidative stress in bone remodeling: role of antioxidants. Clin Cases Miner Bone Metab. 2017; 14(2): 209-16.
7. Khoubnasabjafari M, Ansarin K, Jouyban A. Reliability of malondialdehyde as a biomarker of oxidative stress in psychological disorders. Bio Impacts. 2015; 5(3): 123-7.

8. Sharma T, Islam N, Ahmad J, Akhtar N, Beg M. Correlation between bone mineral density and oxidative stress in postmenopausal women. Indian J Endocrinol Metab. 2015; 19(4): 491-7.

9. Fitri LE, Iskandar A, Sardjono TW, Erliana UD, Rahmawati W, Candradikusuma D. Plasma glutathione and oxidized glutathione level, glutathione/oxidized glutathione ratio, and albumin concentration in complicated and uncomplicated falciparum malaria. Asian Pac J Trop Biomed. 2016; 6(8): 646-50.

10. Jones DP. Redox potential of GSH/GSSG couple: assay and biological significance. Meth Enzymol. 2002; 348: 93-112. 